\title{
DETONATION AND TRANSITION TO DETONATION IN PARTIALLY WATER-FILLED PIPES
}

\author{
Neal P. Bitter and Joseph E. Shepherd \\ Explosion Dynamics Laboratory \\ California Institute of Technology \\ Pasadena, California 91125 \\ Email: nbitter@caltech.edu
}

\section{ABSTRACT}

Detonations and deflagration-to-detonation transition (DDT) are experimentally studied in horizontal pipes which are partially flled with water. The gas layer above the water is stoichiometric hydrogen-oxygen at 1 bar. For detonation cases, ignition and transition occur outside of the water-filled section. For DDT cases, ignition and transition occur over the surface of the water. Pressure and hoop strain are measured incrementally along the pipe, with pressure transducers located both above and below the water. The detonation wave produces an oblique shock train in the water, and the curvature of the pipe is seen to focus the shocks at the bottom, resulting in peak pressures that are 4.6 times higher than the peak detonation pressure. Such pressure amplification is observed for water depths of 0.25, 0.5, 0.75, 0.87, and 0.92 pipe diameters. For a water depth of 0.5 diameters, pressure is also recorded at several circumferential locations in order to measure the shock focusing phenomenon. Peak hoop strains are found to decrease with increasing water depth, and transition to detonation is seen to occur for water depths as high as 0.92 pipe diameters.

\section{NOMENCLATURE}

a Mean pipe radius

i Sound speed of water

$\checkmark$ Inner diameter of pipe

F. Elastic modulus

$h$ Depth of water layer

$k$ Thermal conductivity of pipe
$P_{C J} \quad$ Chapman-Jouguet pressure

$\mathrm{t}$ Time

t' Pseudo-time $t^{\prime}=x / U-t$

U Detonation velocity

$\mathrm{x}$ Spatial coordinate along pipe axis

$\mathrm{X}$ Distance from igniter $(\mathrm{m})$

$\delta \quad$ Pipe wall thickness

$\varepsilon \quad$ Hoop strain

$\varepsilon_{C J} \quad$ Static hoop strain based on Chapman-Jouguet pressure

$\kappa$ Thermal diffusivity of pipe $\frac{k}{\rho_{s} C_{p}}$

$\lambda$ Detonation cell size

$v$ Poisson's ratio

$\omega_{n} \quad$ Natural frequency of pipe in axisymmetric breathing mode

$\Phi$ Dynamic loading factor

$\rho_{s}$ Density of pipe

$\sigma$ Surface tension

$\tau$ Time constant of oblique wave oscillations

$\tau_{d}$ Time constant of pressure decay behind detonation

\section{INTRODUCTION}

Explosions in piping systems are a concern in any application involving transport or handling of combustible gases. In particular, there is a danger that an explosion might produce a detonation wave, resulting in a combination of high pressure and fast energy release that may deform or possibly rupture the pipe. Numerous studies have investigated transition to detonation in piping systems, considering the gas-phase fluid mechanics and chemistry $[1,2]$ as well as the structural response of the pipes [3]. 
Considerably less work has been conducted regarding explosions in pipes that are partially filled with liquid or particulate matter. This situation arises in the nuclear industry, where waste in pipes can release hydrogen and nitrous oxide, forming a combustible gas layer over a liquid or viscous slurry [4].

Detonations in piping systems featuring vertical columns of water have been investigated both experimentally and computationally [5]. However, because the water column is vertical, the interaction between the detonation wave and the liquid is confined to a single location. Detonations over horizontal water layers are in some ways more interesting, since the water and the detonation interact at every location.

Several researchers have studied interactions between shock or detonation waves and horizontal liquid surfaces. Borisov photographed detonations passing over liquid surfaces for several different liquids, and was able to observe the wave reflections below the water as well as the breakup of the gas-liquid interface [6]. More recently, Teodorczyk and Shepherd [7] used both shadowgraph and direct photography to study shock waves (Mach numbers 1.3 - 2.4) passing over water layers in a square channel. The photographs were used to investigate the growth rates of surface waves and spray layers behind the shock wave. Akbar and Shepherd [8] recorded high speed video of DDT over water layers in a square channel and also measured pressures and strains for DDT over water layers in a round pipe. The present study extends the work in [8], seeking to more completely understand how water layers in cylindrical pipes affect the gas dynamics of detonations and DDT, the fluid dynamics in the water, and the structural loading of the piping system.

\section{Experimental Setup}

The test specimen consists of two schedule 40 stainless steel pipes with flanges on both ends, as shown in Fig. 1. Dimensions and material properties of the pipes are provided in Table 1. The pipes are joined together by a blockage element (see inset, Fig. 1) with blockage heights $h$ of $0,0.25,0.5,0.75,0.87$, or 0.92 pipe diameters (denoted $d$ ). The pipe section to the left of the blockage in Fig. 1 has a port through which water can be added, so that the left tube is partially water-filled while the right tube contains only the gas mixture. Two modes of ignition are employed in this experiment. For detonations, ignition is achieved using a spark plug at the end of the right-hand tube, with a $0.3 \mathrm{~m}$ Shchelkin spiral promoting transition to detonation, as depicted in Fig. 1. The detonation wave then passes through the blockage element and over the surface of the water. For DDT, the spark plug is relocated to the port labeled P9 in Fig. 1, and the Shchelkin spiral is removed. Transition to detonation (or lack thereof) can then be observed over the water surface.

Piezo-electric pressure transducers (PCB model 113B22) and strain gauges (Vishay PG, model CEA-09-250-UN) are mounted incrementally along the tube. Pressure is measured at

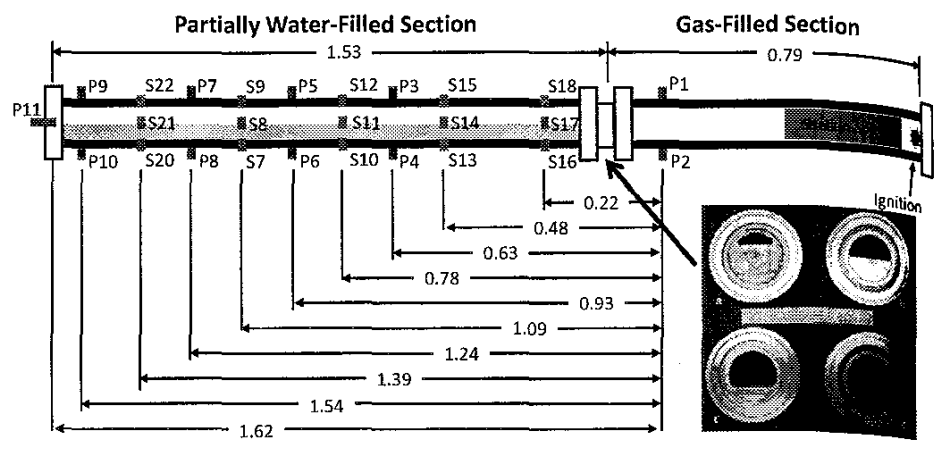

FIGURE 1. Schematic of test setup (dimensions in meters). Strain gauges (S7-S22) are oriented in the hoop direction.

TABLE 1. Material properties and dimensions of schedule 40, stainless steel pipe.

\begin{tabular}{|l|c|c|}
\hline Property & Units & Value \\
\hline Total length & $\mathrm{m}$ & 2.32 \\
\hline Length of water-filled section & $\mathrm{m}$ & 1.53 \\
\hline Length of gas-filled section & $\mathrm{m}$ & 0.79 \\
\hline Outer diameter & $\mathrm{mm}$ & 60.3 \\
\hline Inner diameter & $\mathrm{mm}$ & 52.5 \\
\hline Wall thickness $\delta$ & $\mathrm{mm}$ & 3.9 \\
\hline Total gas volume & $\mathrm{L}$ & 5.2 \\
\hline Elastic Modulus $E$ & $\mathrm{GPa}$ & 193 \\
\hline Density $\rho_{s}$ & $\mathrm{~kg} / \mathrm{m}^{3}$ & 8040 \\
\hline Poisson's ratio $v$ & - & 0.3 \\
\hline Thermal expansion coefficient & $K^{-1}$ & $16.9 \times 10^{-6}$ \\
\hline
\end{tabular}

both the top and bottom of the tube at five locations, as well as at the endwall. Hoop strain is measured at five axial locations along the pipe; at each location three strain gauges are mounted at the bottom, side, and top of the tube. Data were recorded by simultaneous sampling of all channels at a rate of $1 \mathrm{MHz}$.

The gas mixture for all shots was stoichiometric $\mathrm{H} 2-\mathrm{O} 2$ at 1 bar and $300 \mathrm{~K}$. Relevant properties of the mixture are listed in Table 2. Prior to each shot, the test section was evacuated to less than 100 mTorr, after which the fuel and oxidizer were added by partial pressures. After filling, the gases were mixed by running a circulation pump for 5-10 minutes. For shots with water, the pressure after addition and mixing of the fuel and oxidizer was initially kept below the target ignition pressure of 1 bar. Water was then added until the pressure reached 1 bar, after which the mixture was ready for ignition. 
TABLE 2. Thermodynamic properties of gas mixture.

\begin{tabular}{|c|c|c|}
\hline Property & Units & Value \\
\hline Pre-shot pressure & $\mathrm{kPa}$ & 100 \\
\hline Pre-shot temperature & $\mathrm{K}$ & 300 \\
\hline $\mathrm{H} 2$ mole fraction & - & $2 / 3$ \\
\hline $\mathrm{O} 2$ mole fraction & - & $1 / 3$ \\
\hline CJ Speed $U_{C J}$ & $\mathrm{~m} / \mathrm{s}$ & 2834 \\
\hline CJ Pressure $P_{C J}$ & $\mathrm{MPa}$ & 1.87 \\
\hline CJ Reflection Pressure & $\mathrm{MPa}$ & 4.57 \\
\hline Detonation cell width [9] & $\mathrm{mm}$ & 1.39 \\
\hline
\end{tabular}

\section{Results}

Three sets of data were obtained in order to investigate three effects. In the first data set, a Shchelkin spiral was used to promote transition to detonation outside of the water-filled section. This was done for water heights of $h / d=0,0.25,0.50,0.75$, 0.87 , and 0.92 . In the second data set, a constant water height of $h / d=0.50$ was used and the pipe was rotated in increments of $15^{\circ}$ to obtain measurements of pressure vs. $\theta$. For the third data set, the Shchelkin spiral was removed and the mixture was ignited over the water surface for various water heights.

\section{Detonations}

Effects Below the Water Fig. 2 shows baseline pressure traces of a detonation with no water in the test section. The ordinate $X$ marks the distance from each pressure transducer to the igniter. The bottom pressure trace shows partial reflection of the detonation off of the blockage element, which is located at $X=0.79 \mathrm{~m}$. The peak detonation pressures are typically 1.5 1.6 $\mathrm{MPa}$, about $17 \%$ below the CJ pressure of $1.87 \mathrm{MPa}$. This difference is not unusual in experiments involving unsupported detonations [10]; it is the result of non-ideal effects including the cellular structure of the detonation, boundary layer growth, heat Iransfer, and turbulence [11, 12].

Fig. 3 shows pressure traces for a water depth of $h / d=0.50$. An oblique shock train following the detonation wave produces a series of pressure spikes below the surface of the water; a simplified schematic of this wave train is depicted in Fig. 4. In this diagram the detonation velocity is taken to be greater than the liquid sound speed, as is the case in the present experiments. The incident detonation produces an initially compressive wave in the water, which alternates between compression and expansion as it reflects off of the water surface and the bottom of the pipe. Such wave patterns below the water have been observed photographically in both [6] and [8].

Surface Disturbances As drawn in Fig. 4, the water surface breaks up behind the detonation wave. Motion of the

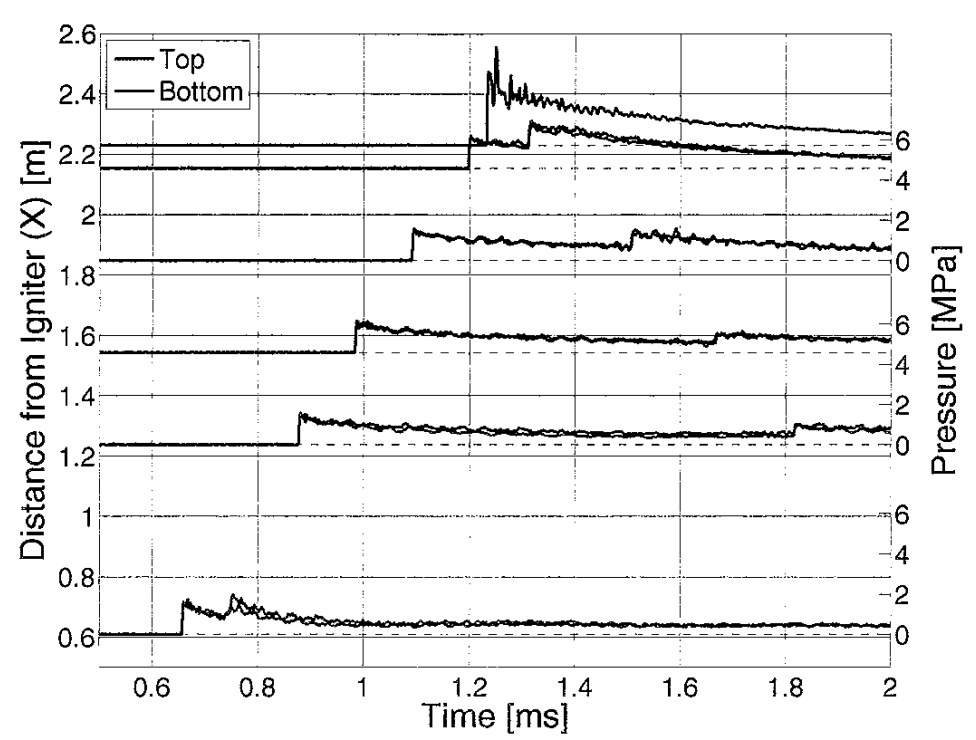

FIGURE 2. Pressure traces for a detonation with no water in the test section.

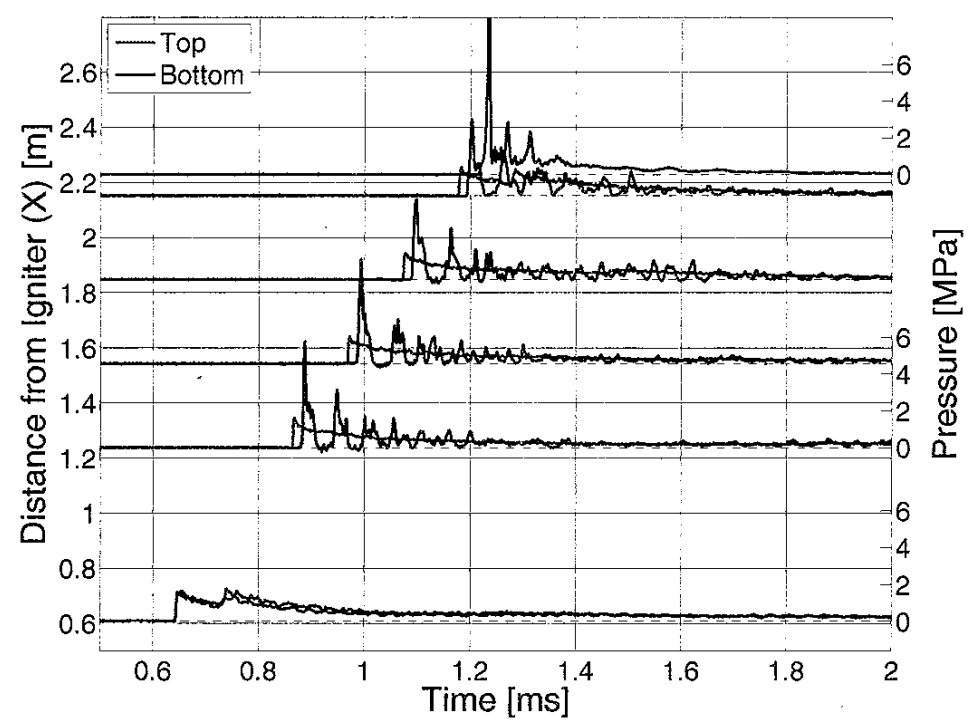

FIGURE 3. Pressure traces for a detonation with water depth $h / d=$ 0.50 .

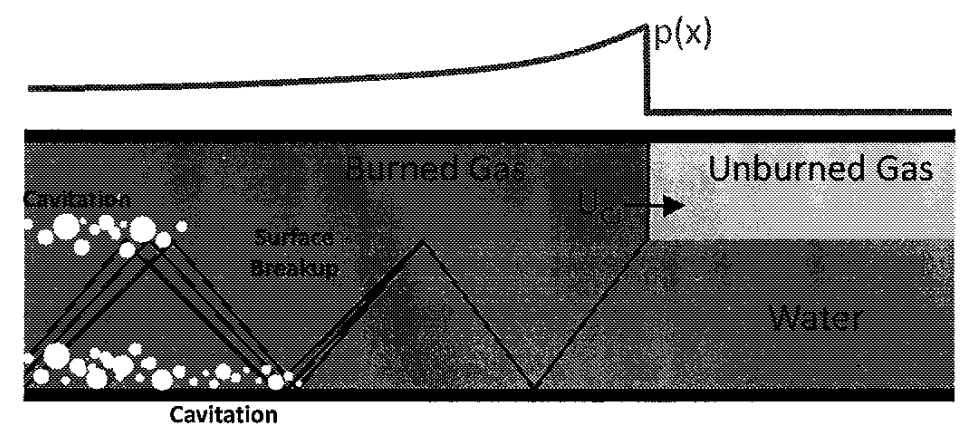

FIGURE 4. Two dimensional wave diagram for a detonation with a wave speed greater than the sound speed of water. 
surface comes from two sources. First, the Kelvin-Helmholtz instability due to the tangential velocity discontinuity at the gasliquid interface causes growth of small scale disturbances, which produces small droplets that are then entrained by the gas flow. Second, the sudden application of a pressure load results in the development of surface waves. It is of interest to estimate the rate of surface motion due to these two effects and compare it with the speed of the detonation wave.

Consider first the shear instability caused by the tangential velocity discontinuity. A linear stability analysis (see, for example, Drazin and Reid [13]) with the inclusion of surface tension produces the following relation between the Laplace transform parameter $s$ and the streamwise wavenumber $k$ :

$$
\begin{aligned}
s & =i k \frac{\rho_{g} U}{\rho_{g}+\rho_{w}} \\
& \pm \sqrt{\frac{k^{2} \rho_{w} \rho_{g} U^{2}}{\left(\rho_{w}+\rho_{g}\right)^{2}}-\frac{k^{2}}{\rho_{w}+\rho_{g}}\left[\frac{g\left(\rho_{w}-\rho_{g}\right)}{k}+k \sigma\right]}
\end{aligned}
$$

The spanwise wavenumber has been set to zero in accordance with Squire's theorem. Here $U$ is the gas velocity behind the detonation, $\rho_{w}$ is the water density, $\rho_{g}$ is the burned gas density $(0.88$ $\left.\mathrm{kg} / \mathrm{m}^{3}\right), \mathrm{g}$ is gravitation, and $\sigma$ is the surface tension $(72 \mathrm{mN} / \mathrm{m})$. For these parameters, instability occurs if $U>7.8 \mathrm{~m} / \mathrm{s}$, which is certainly satisfied since $\mathrm{CJ}$ theory predicts $U=1290 \mathrm{~m} / \mathrm{s}$. For $U=$ $1290 \mathrm{~m} / \mathrm{s}$, the most amplified wavelength is about $75 \mathrm{~nm}$, which is likely below the range of validity of this model since at such small scales the surface tension model is no longer appropriate and the gas-phase boundary layer is large compared to the disturbances. Nevertheless, this model shows that at short times the shear instability significantly amplifies only small disturbances; for example, at $100 \mu \mathrm{s}(0.28 \mathrm{~m})$ behind the detonation, disturbances of wavelength greater than $5 \mathrm{~mm}$ have not yet doubled in size. As a result, no large-scale motion of the surface is expected at times on the order of $100 \mu s$; rather, the surface will break up into small (millimeter scale or smaller) droplets which will then be entrained in the flowing gas, forming a mist layer.

The growth rate of the mist layer is more difficult to estimate analytically, but experiments by Teodorczyk and Shepherd [7] show that for shock waves over water, the mist layer grows approximately linearly in time and the growth rate is nearly independent of the shock Mach number (for Mach numbers in the range 1.3-2.3). They measured growth rates on the order of 10 $\mathrm{m} / \mathrm{s}$, more than two orders of magnitude smaller than the detonation speed of $\sim 2800 \mathrm{~m} / \mathrm{s}$ for the current experiments.

The second source of surface motion is the suddenly applied pressure. Treating the water acoustically, the vertical velocity of the free surface $\Delta u$ immediately after the passage of the detona- tion wave can be approximated by:

$$
\Delta u=\frac{\Delta p}{\rho_{w} c_{w}} \approx 1.25 m / s
$$

where $\Delta p$ is the pressure jump across the detonation and $c_{w}$ is the sound speed in water. Thus in the first $100 \mu \mathrm{s}(0.28 \mathrm{~m})$ behind the detonation, the surface deflection due to the applied pressure is only about $0.1 \mathrm{~mm}$.

Based on the above calculations, surface deformation and mist layer development are not expected to be important until more than $100 \mu \mathrm{s}$ after the detonation. This is consistent with the experimental observations reported in [6-8]. The work of Akbar and Shepherd [8] is particularly useful since the dimensions, liquid, and detonation speeds from that experiment are quite close to those in the present study. Hence the distance behind the detonation at which surface breakup becomes appreciable is probably similar in the present experiments. Using high speed video from [8], this distance was estimated to be between 0.3 and 0.4 $\mathrm{m}$, which corresponds to about $100-140 \mu \mathrm{s}$.

Cavitation Akbar and Shepherd [8] also observed photographically that expansion waves below the water can be strong enough to bring the fluid into tension, resulting in cavitation. This typically produces a cavitated layer along the bottom of the pipe as well as at the surface of the water, as drawn in Fig. 4. The cavitation bubbles at the bottom of the pipe form after the passage of the first expansion wave and persist until the next compression wave arrives, at which point the vapor bubbles collapse. These phenomena are clearly visible in the pressure traces of Fig. 3. The incident compression wave appears as a pressure spike followed by a plateau of nearly constant pressure, which rapidly drops off upon arrival of the expansion wave. The pressure at the bottom of the pipe then remains at a constant, low pressure which is (within the capabilities of the pressure transducers) equal to the vapor pressure, signifying cavitation. Upon arrival of the next compression wave, the vapor bubbles collapse and the pressure rises once again. This process repeats itself, resulting in a series of pressure spikes separated by troughs of low, constant pressure.

It is well known that the collapse of cavitation bubbles can produce very high local pressures. Inaba and Shepherd [14] investigated the appearance and collapse of cavitation bubbles in vertical, water-filled tubes and obtained results that are quite similar to those shown in Fig. 3. They applied an impulsive pressure loading to the water at the top of their vertical tube; the resulting wave then reverberated back and forth along the axis of the tube, switching between compression and expansion with every subsequent reflection from the top end of the tube. At the bottom of the tube, they recorded pressure traces exhibiting periodic plateaus of high pressure separated by regions of low, constant pressure 
due to cavitation (similar to Fig. 3). They also observed peak pressures due to bubble collapse that were nearly three times the pressure of the incident wave. In view of these findings, it would not be surprising if the second pressure spike in Fig. 3 demonstrated high pressures due to vapor collapse. However, vapor collapse cannot be responsible for the magnitude of the first pressure spike, since the initial wave in the water is compressive and vapor has not yet formed. As will be shown in the next section, the high pressures in the initial wave are caused instead by the curved walls of the pipe. Furthermore, the second and third pressure peaks in Fig. 3 have waveforms similar to the first peak, which is evidence that shock wave reflection and the curved pipe walls dictate the shape of the pressure trace, while vapor collapse plays a relatively smaller role.

Wave Reflection Model The simplified 2D representation of the flow shown in Fig. 4 is sufficient to explain some (but not all) of the features of the pressure traces. For instance, the period of the observed pressure spikes is very close to the value $\tau \approx 4 h / c$ that would be expected for acoustic waves in a $2 \mathrm{D}$ channel. For example, when $h / d=0.50$, the expected period is $\tau=68 \mu \mathrm{s}$ and the measured periods are typically $60-70$ $\mu s$. Other features of the pressure traces, however, are governed by three-dimensional effects involving the curvature of the pipe wall.

The most striking of these three dimensional effects is that the peak pressure below the water is much greater than would be expected from a two dimensional model. For example, consider the prediction one would make if the pipe were modeled as a $2 \mathrm{D}$ planar channel instead of a circular pipe. For the given test conditions, the detonation pressure satisfies $\frac{\Delta P}{\rho c U} \sim 0.0004<<1$, meaning that the oblique shocks in the water are merely acoustic waves. Since the detonation pressure $(\sim 1.87 \mathrm{MPa})$ is much larger than the initial pressure $(\sim 0.1 \mathrm{MPa})$, the expected peak pressure based on the 2D planar model would then be about twice the peak detonation pressure. In contrast, the pressures observed in this experiment are 4-6 times the peak detonation pressure.

These high pressures are caused by the curved walls of the pipe. To further examine this effect, the acoustic waves in the water were modeled using the linear wave equation:

$$
\frac{\partial^{2} p}{\partial t^{2}}=c^{2}\left(\frac{\partial^{2} p}{\partial r^{2}}+\frac{1}{r} \frac{\partial p}{\partial r}+\frac{1}{r^{2}} \frac{\partial^{2} p}{\partial \theta^{2}}+\frac{\partial^{2} p}{\partial x^{2}}\right)
$$

The pressure loading of the detonation wave, denoted $p_{d}(x, t)$, can be well approximated as a traveling wave which is a function only of the composite variable $t^{\prime}=x / U-t$, where $\mathrm{x}$ is the axial coordinate and $U$ is the detonation velocity. The solution is then expected to be of the form $p=p(x / U-t, r, \theta)$, which means that the problem can be greatly simplified by transforming into a frame of reference that is fixed to the detonation wave. After making this transformation, the 3D equation above is reduced to a $2 \mathrm{D}$ wave equation with a modified wave speed:

$$
\frac{\partial^{2} p}{\partial t^{\prime 2}}=\left(\frac{c^{2}}{1-\frac{c^{2}}{U^{2}}}\right)\left(\frac{\partial^{2} p}{\partial r^{2}}+\frac{1}{r} \frac{\partial p}{\partial r}+\frac{1}{r^{2}} \frac{\partial^{2} p}{\partial \theta^{2}}\right)
$$

Only the supersonic case of $U>c$, which is relevant to the present situation, is considered here. Equation 4 is subject to the initial and boundary conditions:

$$
\begin{array}{r}
\left.\frac{\partial p}{\partial t^{\prime}}\right|_{t^{\prime}=0}=0 \\
\left.\frac{\partial p}{\partial r}\right|_{r=a}=0 \\
p(\theta=0)=p(\theta=\pi)=p_{d}\left(t^{\prime}\right)
\end{array}
$$

where $p_{d}$ is the detonation pressure. By applying a boundary condition at $\theta=0$ and $\theta=\pi$, it is implicitly assumed that deformation of the free surface is small. As discussed previously, this is only valid for a short distance behind the detonation; for the test conditions in this experiment the time over which this assumption is valid was previously estimated to be about $100 \mu \mathrm{s}$.

The detonation pressure $p_{d}\left(t^{\prime}\right)$ was modeled by fitting the experimental data with an equation of the form:

$$
p_{d}\left(t^{\prime}\right)=p_{2}+\left(p_{1}-p_{2}\right) \exp \left(-\frac{t^{\prime}}{\tau_{d}}\right)
$$

where $p_{1}$ is the detonation's peak pressure, $p_{2}$ is limit toward which the pressure behind the detonation decays, and $\tau_{d}$ is the time constant of the decay. To solve the system of Eqs. 4-5, it is convenient to make a change of variables $\bar{p}=p-p_{d}\left(t^{\prime}\right)$ so that the boundary conditions become homogeneous. The wave equation then becomes:

$$
\frac{\partial^{2} \bar{p}}{\partial t^{2}}=\bar{c}^{2}\left(\frac{\partial^{2} \bar{p}}{\partial r^{2}}+\frac{1}{r} \frac{\partial \bar{p}}{\partial r}+\frac{1}{r^{2}} \frac{\partial^{2} \bar{p}}{\partial \theta^{2}}\right)+\frac{\partial^{2}}{\partial t^{2}} p_{d}\left(t^{\prime}\right)
$$

Here $\bar{c}^{2}=c^{2} /\left(1-c^{2} / U^{2}\right)$ is the effective wave speed produced by the transformation to wave-fixed coordinates. The nonhomogeneous term in Eq. 7 can be expanded as a sum of the homogeneous eigenfunctions, resulting in a solution is of the form:

$$
\begin{aligned}
p\left(r, \theta, t^{\prime}\right) & =\bar{p}\left(r, \theta, t^{\prime}\right)+p_{d}\left(t^{\prime}\right) \\
& =\sum_{m=1}^{\infty} \sum_{n=1}^{\infty} A_{m n}\left(t^{\prime}\right) J_{n}\left(\lambda_{m n} r\right) \sin (n \theta) \cos \left(\lambda_{m n} \bar{c} t^{\prime}\right)+p_{d}\left(t^{\prime}\right)
\end{aligned}
$$


where $J_{n}$ is the $\mathrm{n}^{t h}$ order Bessel function of the first kind. To adequately resolve pressure discontinuities in the solution, a large number of terms is required in the double summation. Solutions were compared using up to 200 terms in each summation; about 100 terms was found to provide a sufficient level of convergence.

Several representative pressure contours are shown in Fig. 5. The incident wave initially moves parallel to the wall, which acts like a gradually steepening cylindrical wedge. As discussed in [15], a Mach reflection occurs initially, as is required to satisfy the wall-tangency condition. In frames 1-2, the reflected branch of the Mach reflection forms a shock wave sweeping along the pipe wall; this shock will hereafter be called the wall shock. As the wall continues to steepen relative to the incident wave, the Mach stem decreases in length and the wall shock increases in strength until the Mach reflection becomes a regular reflection.

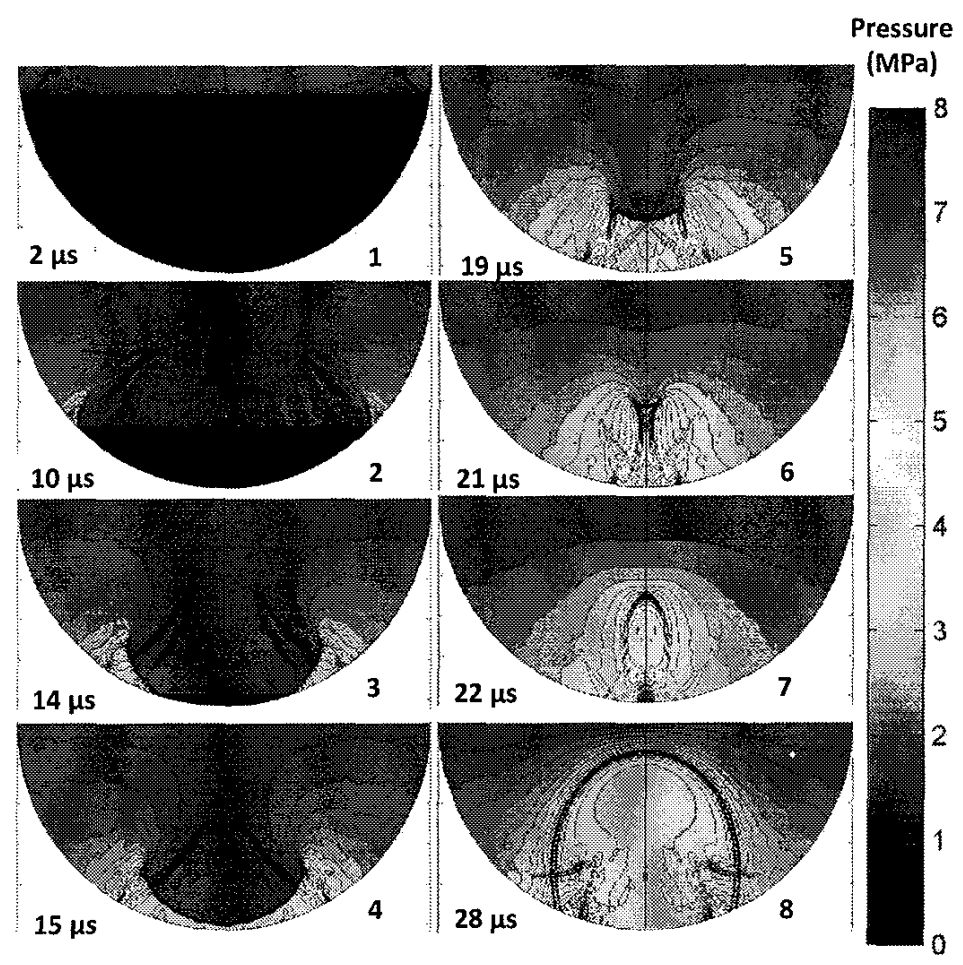

FIGURE 5. Pressure contours from a series solution of the equivalent 2D transient problem.

Near the bottom of the pipe (frames 3-4), the point of intersection between the incident shock and the pipe wall moves more quickly than the wall shocks. This happens because the incident shock travels at a constant speed $U_{I}$ while the point of intersection with the wall moves with speed $U_{I} / \sin \phi$, where $\phi$ is the local wall angle relative to the incident shock. As the incident shock approaches the bottom of the pipe, $\phi$ tends to $90^{\circ}$ so the intersection point outruns the wall shocks.

After the incident wave has completely reflected off of the bottom of the pipe, its regular reflection travels away from the bottom of the pipe while the wall shocks continue to sweep in ward (frames 4-6). In frames 7-8, the two wall shocks cross and merge to form a single, horseshoe-shaped, expanding shock wave which decreases in strength as it expands. The solution is valid until this expanding shock reflects off of the free surface. At this point, the model predicts negative pressures, which will lead cavitation.

Peak pressures during the shock reflection process occur at the bottom of the pipe and at a point interior to the fluid; at both locations, the maximum pressure is reached when the two wall shocks merge (frame 7). The predicted peak pressure at the base of the pipe is $7.8 \mathrm{MPa}$, which is about $30 \%$ greater than the max imum pressures observed in experiment for this water height However, this peak pressure only lasts for a few microseconds and is confined to a very small region; it is likely that the finite width and response time of the pressure transducer prevent the actual peak pressure from being resolved experimentally.

In the above results, the interaction between the incident shock and the pipe wall produced only simple Mach reflections and regular reflections. The situation can be much more complicated for stronger incident shocks; additional reflection states become possible, which in general depend on shock strength, the pipe's radius of curvature, and the wall angle at at the surface of the water [15]. This means that different behavior could be expected for different water depths. However, in the present case (and in most practical cases involving detonations over water) the shocks are very weak, so only the Mach reflection and regular reflection can occur [15]. A more detailed discussion of the various shock reflection states that can take place along the pipe wall is available in $[15,16]$. In addition, an excellent experimental visualization and confirmation of this effect can be found in [16], where the analogous problem of a weak planar shock wave (Mach number 1.04) reflecting off of a cylindrical surface is examined using schlieren photography.

Pressure traces for other selected water depths of $h / d=$ $0.25,0.75$, and 0.92 are shown in Figs. 6-8 (test data and pressure traces for other water heights not plotted here are available in [17]). The period of the pressure spikes appears to vary proportionally with water depth, as anticipated based on the explanations above. No clear relationship between peak pressure below the water and water depth was found, but peak pressures typically ranged from 5.5-8.0 $\mathrm{MPa}$, giving a ratio of peak pressure to incident pressure in the range of 4-6.

Effects Above the Water Although the effect of the water is much greater at the bottom of the pipe, the influence on the gasdynamics above the water is not insignificant. One effect of the water is that the reflected shock wave is rapidly attentated, with greater attenuation occurring at greater water depths. This is highlighted by overlaying several pressure traces above 


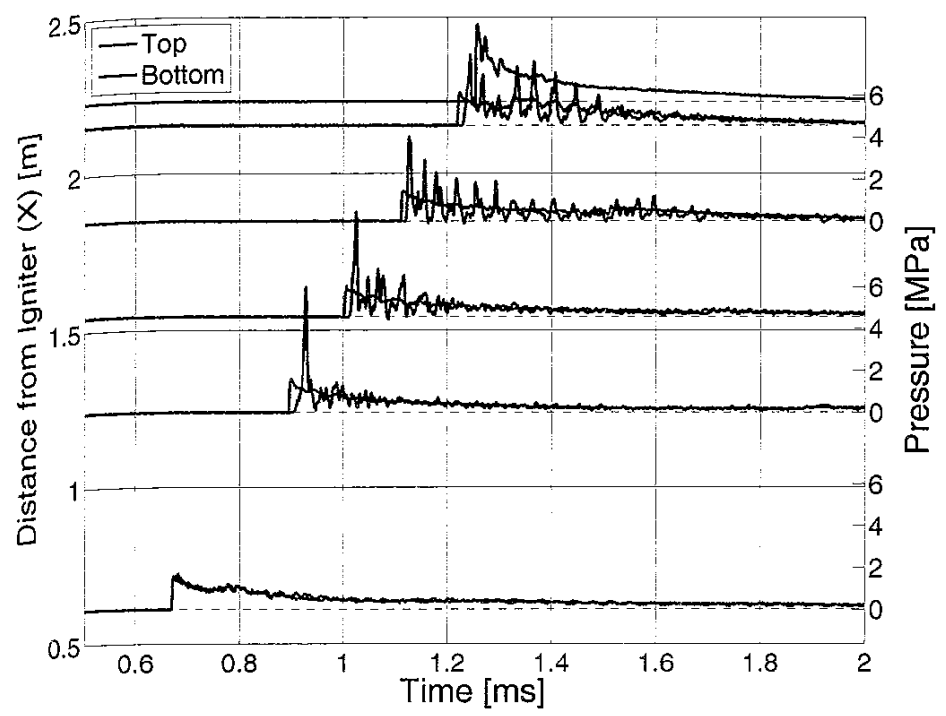

FIGURE 6. Pressure traces for a detonation with $h / d=0.25$.

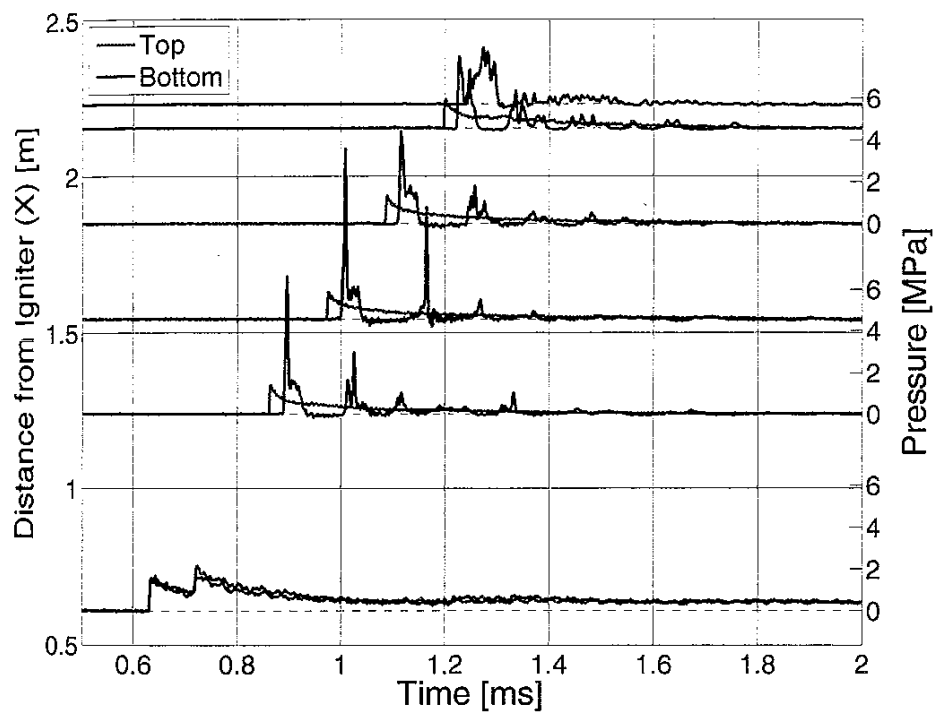

FIGURE 7. Pressure traces for a detonation with $h / d=0.75$.

the water, as shown in Fig. 9.

These traces are recorded $1 \mathrm{~m}$ from the end wall. The passage of the reflected shock at $t \sim 0.95 \mathrm{~ms}$ is only slightly visible when the pipe is half full, and is not detectable for a water height of $h / d=0.87$. The attenuation of the reflected wave is presumably caused by water droplets that are dispersed behind the detonation due to surface breakup. As discussed earlier, droplet dispersion is negligible on the microsecond timescale associated with wave motion below the water, but in Fig. 9 it is clear that droplet dispersion becomes important on the millisecond timescale, which is consistent with the experimental observations of [8]. The water droplets are injected into the gas layer

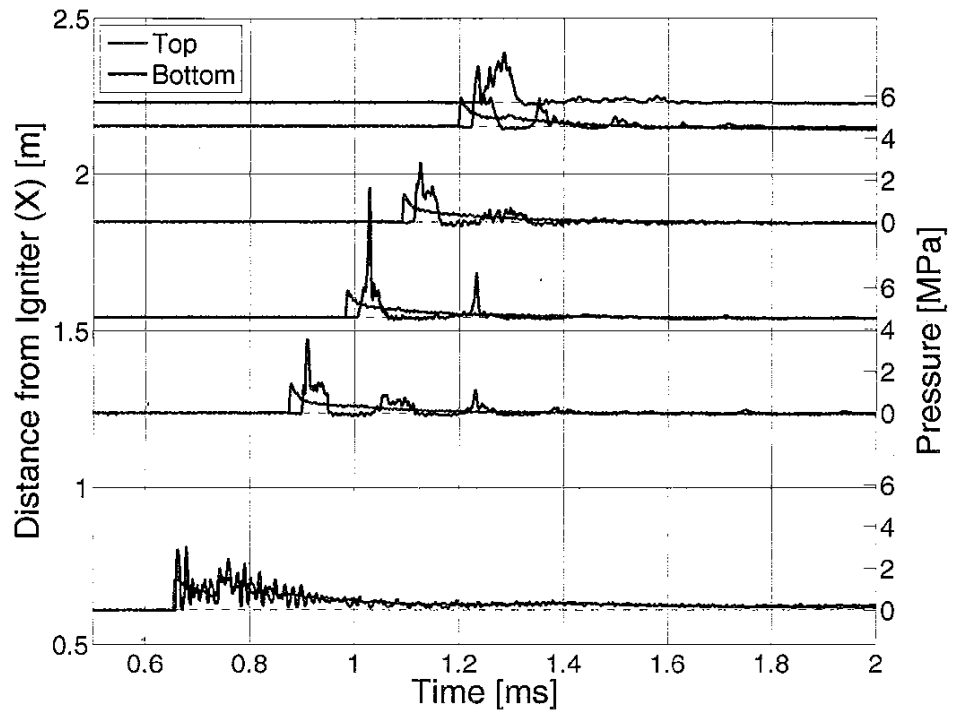

FIGURE 8. Pressure traces for a detonation with $h / d=0.92$. High frequency oscillations on the bottom trace are due to pressure reflections in a very thin layer of water that spilled over from the water-filled section.

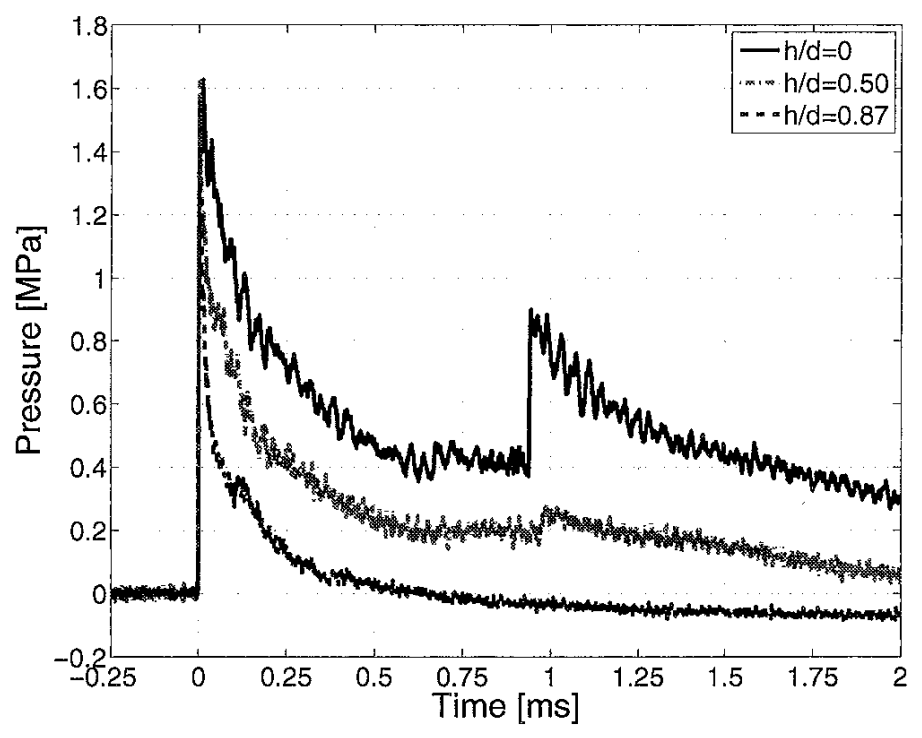

FIGURE 9. Overlaid pressure traces for water heights $h / d=0,0.5$, and 0.87 for transducer P3 (above the water, $1 \mathrm{~m}$ from the pipe's endwall).

with velocities much smaller than the gas velocity, which produces a large momentum sink and hence reduces the pressure behind the detonation. Images recorded in [8] (an experiment that is dimensionally similar to the present setup) show that for thicker gas layers $(\sim 13 \mathrm{~mm})$, the water droplets may not penetrate through the entire gas layer before the passage of the reflected shock. This explains why the attenuation of the reflected shock increases with water depth. 
The second effect of the water (also shown in Fig. 9) is an increased rate of pressure decay behind the detonation. This is again explained by dispersion of water droplets; the large surface area of the many droplets results in rapid heat transfer and a substantial reduction in temperature.

One might expect that so much heat transfer to the water droplets would result in significant evaporation, a pressure increasing mechanism, thereby producing negative feedback and preventing the pressure from falling. Evidently the thermal mass of the scattered water is large enough that the water temperature does not (on average) need to exceed the boiling point in order to cool the burned gases. In fact, the amount of energy needed to cool a unit mass of burned gas (in this case, steam) from the CJ temperature of $\sim 3500 \mathrm{~K}$ to ambient temperature is less than four times the energy needed to boil the same mass of water. Thus if the mass of the dispersed droplets is more than four times the mass of the gas layer, evaporation need not occur at all.

While Fig. 9 shows similar peak detonation pressures for several water heights, the peak pressure above the water does in fact decrease slightly with water depth. This is shown in Fig. 10, where peak pressures (recorded before detonation reflection) are plotted against water height. About a 13\% decrease is observed at the highest water depths, and is likely caused by friction and heat transfer to the water surface and pipe walls.

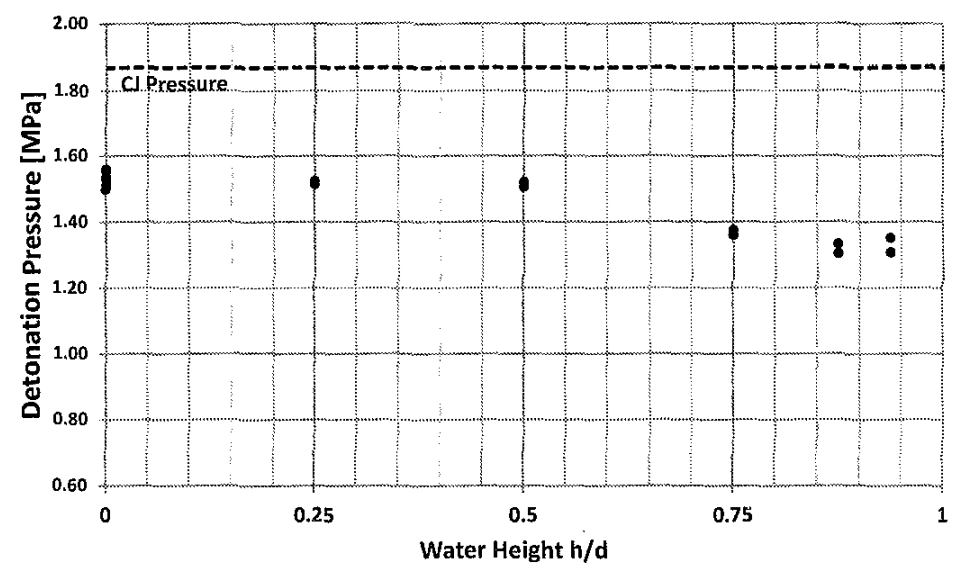

FIGURE 10. Relationship between peak detonation pressure and water height. Peak pressures are recorded above the water, prior to passage of the reflected shock wave. Each data point corresponds to an individual shot.

Despite the modest decrease in peak pressure, the detonation showed no evidence of quenching even at the highest water depth, which corresponds to a gas layer thickness of $\sim 4 \mathrm{~mm}$. It is worth noting that the detonation cell size for stoichiometric H2$\mathrm{O} 2$ is about $1.4 \mathrm{~mm} \mathrm{[9],} \mathrm{somewhat} \mathrm{smaller} \mathrm{than} \mathrm{the} \mathrm{minimum}$ gas layer height tested in this experiment. Other mixtures, such as steam-diluted H2-air or H2-N2O (both relevant in the nuclear industry) can have cell sizes 1-3 orders of magnitude larger, and may thus behave differently at such small gas layer heights.

\section{Pressure Variation with $\theta$}

To study the hypothesized shock focusing effect discussed previously, a constant water depth of $h / d=0.50$ was used and the pipe was rotated from $0^{\circ}$ to $75^{\circ}$ in $15^{\circ}$ increments. Pressure and strain measurements in this setup are typically quite repeatable for detonations initiated with the Shchelkin spiral, which justifies the use of data from multiple shots to investigate the spatial variation of pressure. Contour plots of pressure plotted against $\theta$ and time were constructed for each pressure transducer. A typical plot is shown in Fig. 11, with the bottom of the pipe taken to be $\theta=0$. Two shots were recorded at each angle; one is plotted as a positive angle and the other as a negative angle, so that the degree of symmetry of the contour plot about $\theta=0$ is representative of the repeatability of the data.

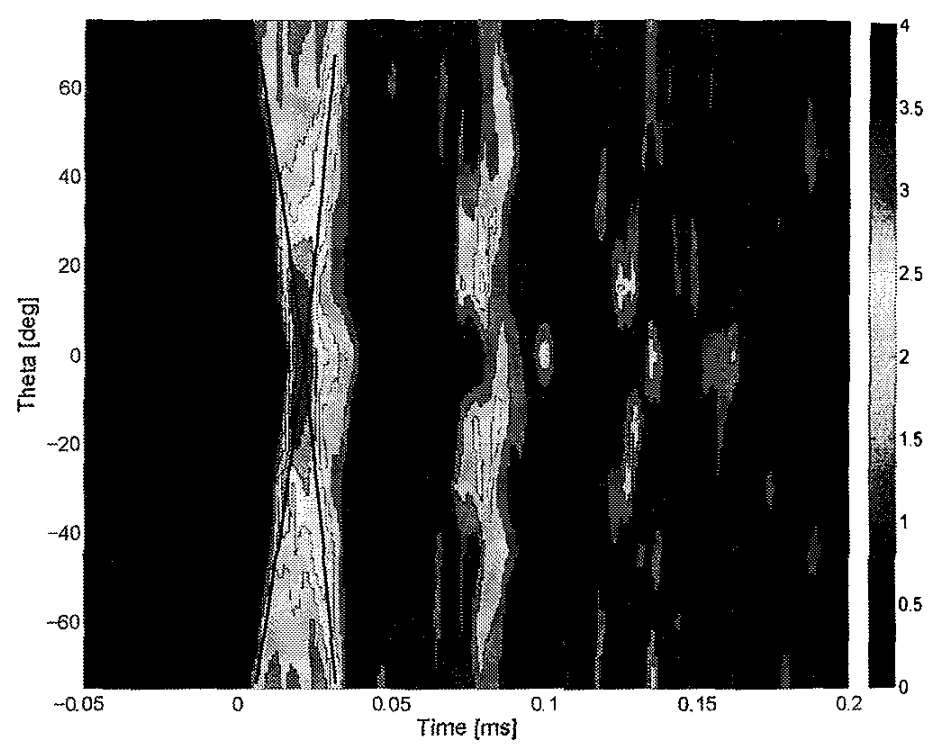

FIGURE 11. Contours of pressure P6 (MPa) against time and $\theta$ for $h / d=0.50$. The bottom of the pipe is denoted $\theta=0$. Two trials were recorded for each angle, with one plotted as $+\theta$ and the other as $-\theta$. Black lines mark the trajectories wall shocks, see Fig. 5.

The resulting contour plots show bands of high pressure followed by bands of low pressure, which correspond to the periodic pressure peaks and troughs in Fig. 3. The curved leading edge of the first pressure band demonstrates the increasing time delay between the passage of the detonation above water and the arrival of the pressure wave at the transducer below water. In the first pressure band, the trajectories of the wall shocks are marked with black lines. At $\theta=90^{\circ}$ (i.e., at the surface of the water), the pressure after the wall shock is equal to the detonation pressure 


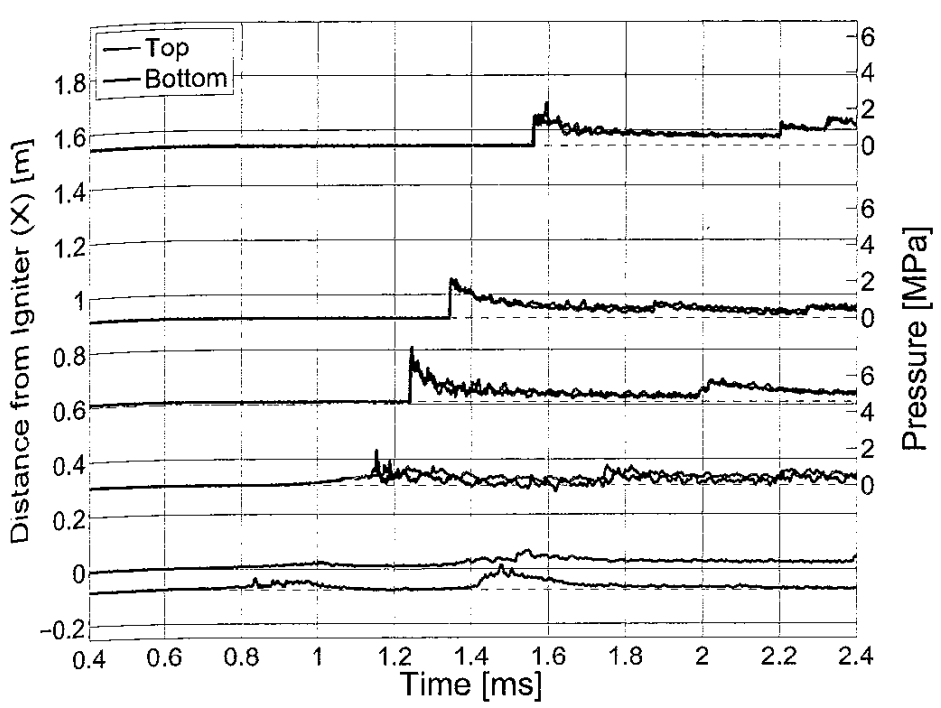

FIGURE 12. Baseline pressure traces for DDT with no water in tube.

above the water. As the wall shock travels towards the base of the pipe, the pressure increases, reaching a maximum at $\theta=0^{\circ}$. After reaching the bottom of the pipe, the wall shocks merge to form the horseshoe-shaped expanding shock that is depicted in Fig. 5, which decreases in strength as it moves toward the free surface.

After this horseshoe-shaped expanding shock strikes the free surface of the water, the behavior becomes much more complicated. In addition to the geometrical complications, cavitation, surface deformation, and surface breakup are presumed to interfere with the reflection of this wave off of the free surface of the water, resulting in a scattered reflected wave. As a result, the second pressure wave is much less distinct, and subsequent waves are hardly distinguishable.

\section{Deflagration to Detonation Transition}

The third effect investigated is transition to detonation over the water surface. Pressure traces from a baseline explosion with no water in the pipe are shown in Fig. 12. DDT occurs between 0.3 and $0.6 \mathrm{~m}$ from the ignition point, and a retonation wave is seen propagating back through the combustion products towards the ignition source and reflecting off of the end wall. After transition, the pressures at the top and bottom of the tube are nearly the same. The peak pressure after transition is typically between 2 and $3 \mathrm{MPa}$, somewhat greater than the CJ pressure, which is typical of the initially overdriven detonations that occur after DDT [11]. The peak pressure then decays towards the CJ value as the wave progresses.

Pressure traces for a water layer of depth $h / d=0.50$ are shown in Fig. 13. In this case, the DDT event occurs very close to $0.3 \mathrm{~m}$ from ignition, where both precompression and a pressure spike due to DDT are observed. At all subsequent transducers,

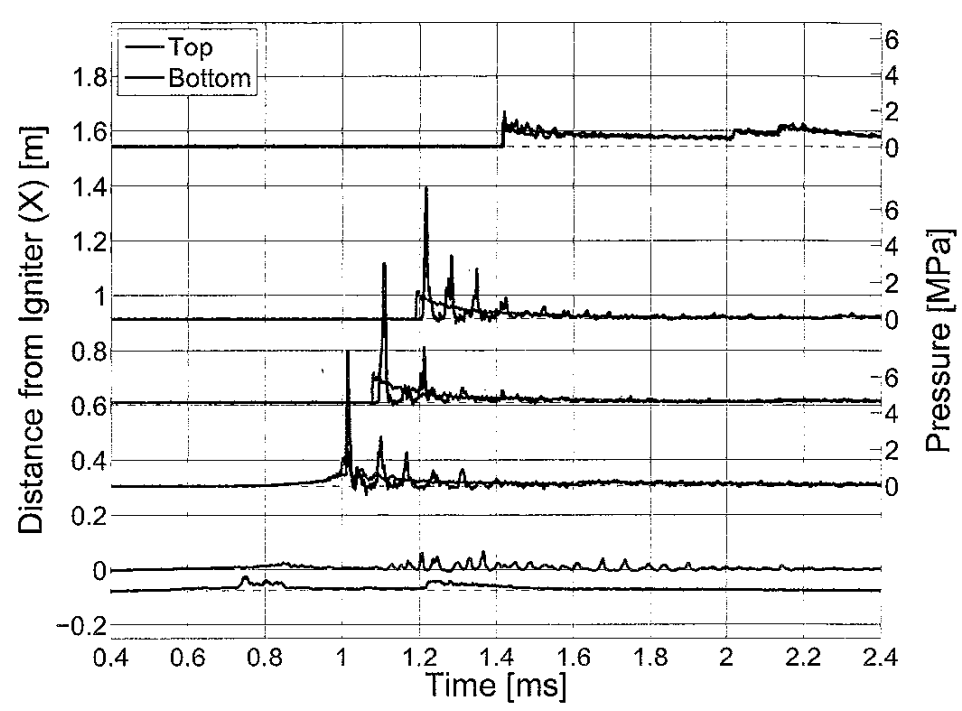

FIGURE 13. Pressure traces for DDT with water depth $h / d=0.50$.

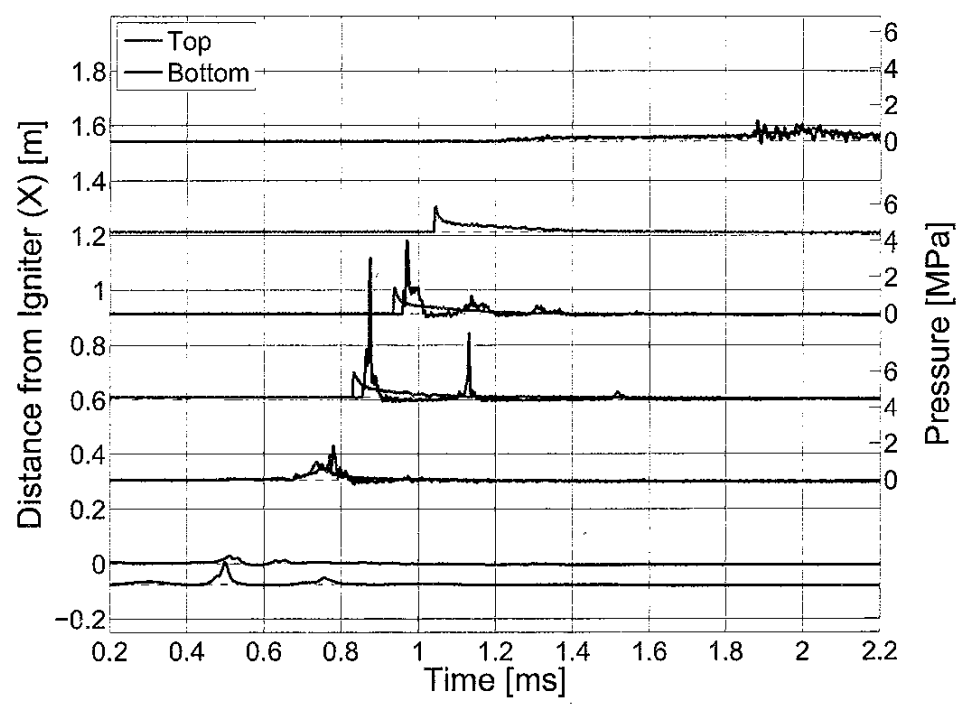

FIGURE 14. Pressure traces for DDT with water depth $h / d=0.92$.

a detonation wave is seen with pressure traces above and below water that resemble those shown earlier for detonations.

Pressure traces for the deepest water layer tested, $h / d=0.92$, are shown in Fig.14. Transition to detonation appears unaffected by the water, but in this case the size of the gas layer is below the critical dimensions for successful propagation of a diffracted detonation. As a result, the detonation fails as it passes from the thin gas layer in the water-filled section to the larger gas-filled section (see Fig. 1). This is evident from the absence of a pressure jump in the top pressure trace, which is located just after the interface between the empty and water-filled sections $(X=1.47$ $\mathrm{m})$. For all other water depths tested, the detonation successfully negotiated this change in area. For $h / d=0.92$, the width of the gas layer is $25 \mathrm{~mm}$ and the height is $4 \mathrm{~mm}$, so the aspect ratio 


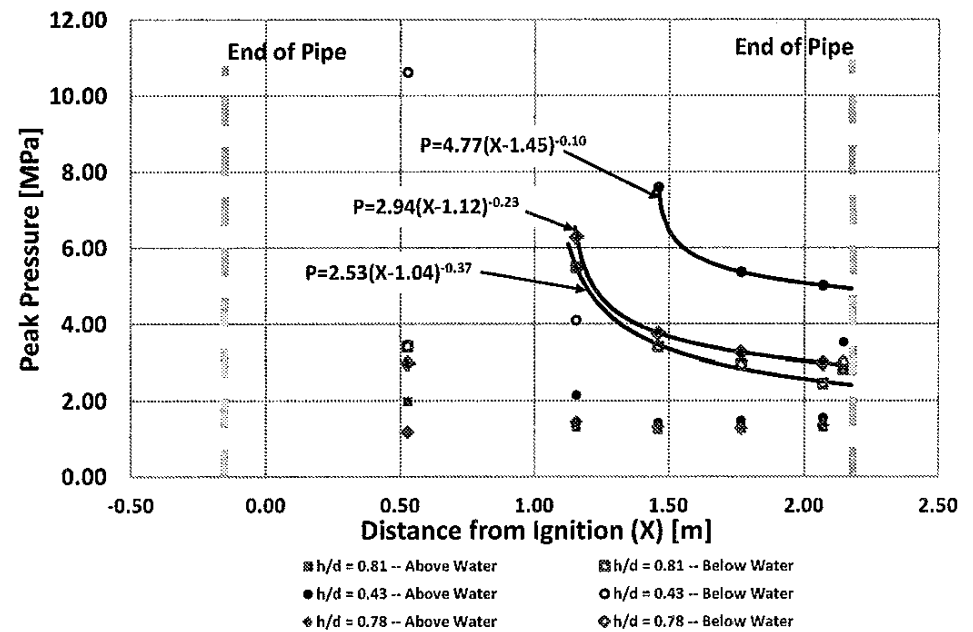

FIGURE 15. Peak pressure below water vs. position for DDT over several water heights.

is about 6. Experiments in [18] reveal that for a square channel with an aspect ratio greater than $\sim 5$, the critical channel height for detonation diffraction is independent of channel width and is equal to $3 \lambda$, where $\lambda$ is the detonation cell size. In this experiment, $3 \lambda=4.2 \mathrm{~mm}$, which is very close to the channel height of $4 \mathrm{~mm}$ at which detonation failure was observed.

The water also has an interesting effect on the decay of the blast wave produced during the DDT event. To examine this effect, the blockage element was removed (see Fig. 1) and both halves of the tube were partially filled with water. The mixture was then ignited at the right-hand side of the tube in Fig. 1, rather than at the left-hand side as before. This was done so that the majority of the pressure transducers would be located after the DDT event rather than before it. The resulting measurements of peak pressure vs. position are plotted in Fig. 15. It appears that the initial blast wave created by the DDT event decays more rapidly as the water depth increases. For comparison, these decay rates are all slower than that of an acoustic cylindrical wave in free space, which decays like $r^{-0.5}$ [19]. This is expected, since confinement reduces the decay rate of the blast wave.

For every shot, the transition distance was between 0.3 and $0.6 \mathrm{~m}$. Since the spatial resolution of the pressure transducers is limited in this experment, the relationship between transition distance and water height could not be more precisely measured.

For all water layers, even as deep as $h / d=0.92$ (a gas layer height of $4 \mathrm{~mm}$ ), transition to detonation was not prevented by the water. Peak pressures also did not change significantly, even at the highest water depths. As discussed previously, the detonation cell size for stoichiometric $\mathrm{H} 2-\mathrm{O} 2$ is smaller than the smallest gas layer tested. Behavior may be different for mixtures with larger cell sizes.

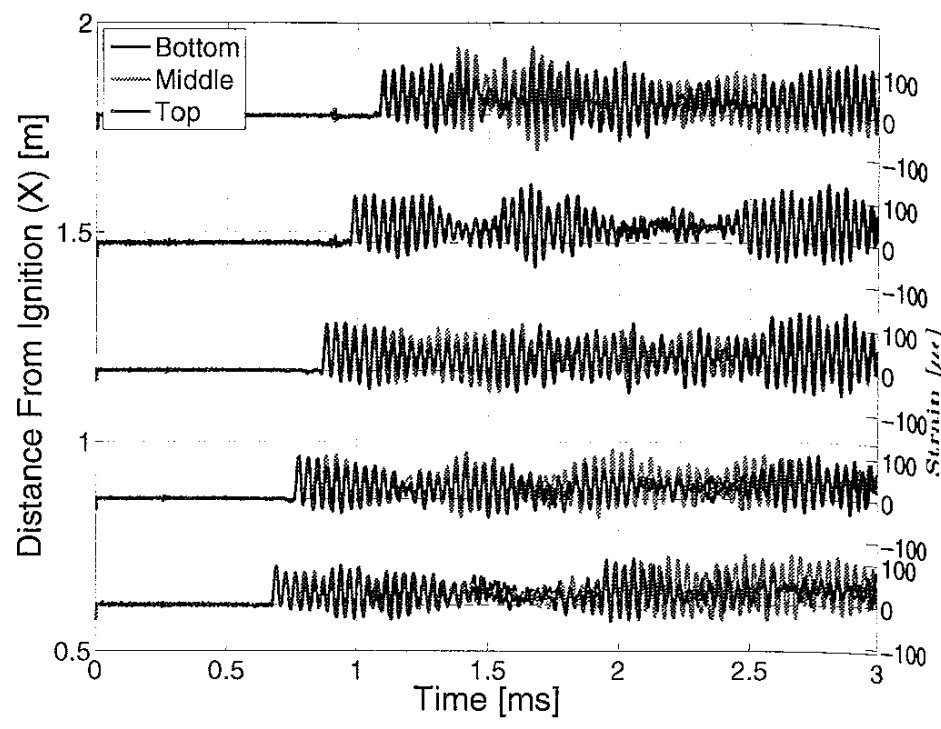

FIGURE 16. Strain traces (hoop direction) for a detonation with no water in pipe.

\section{Structural Loading}

Typical strain traces for a detonation with no water are shown in Fig. 16. The hoop strains at the top, side, and bot. tom of the pipe oscillate in phase at $29 \mathrm{kHz}$, which is equal to the natural frequency of the first (axisymmetric) breathing mode. given by Eq. 9 [20].

$$
\omega_{n}=\frac{1}{2 \pi a} \sqrt{\frac{E}{\rho_{s}\left(1-v^{2}\right)}}
$$

Strain traces for a water layer of height $h / d=0.50$ are shown in Fig. 17. The top, side, and bottom strains are no longer in phase, so the energy of oscillation is no longer concentrated at a single frequency. Furthermore, the hoop strains are damped by the water, as is quantified in Fig. 18 where the dynamic loading factor $\Phi$ is plotted against water height. The dynamic loading factor is defined in this context as the peak strain normalized by the static hoop strain that would occur at the CJ pressure:

$$
\Phi=\frac{\varepsilon_{\max } E \delta}{P_{C J} a}
$$

where $\delta$ is the wall thickness of the pipe. The maximum observed dynamic loading factor for a detonation is about 2.5 with no water, and decreases systematically as the water depth increases. For DDT, the dynamic load factor without water is about 3.5-4.5, and exceeds that of a detonation because the peak pressures are higher. As the water depth increases, the high pressures produced during DDT decay more rapidly (see Fig. 15), and thus 


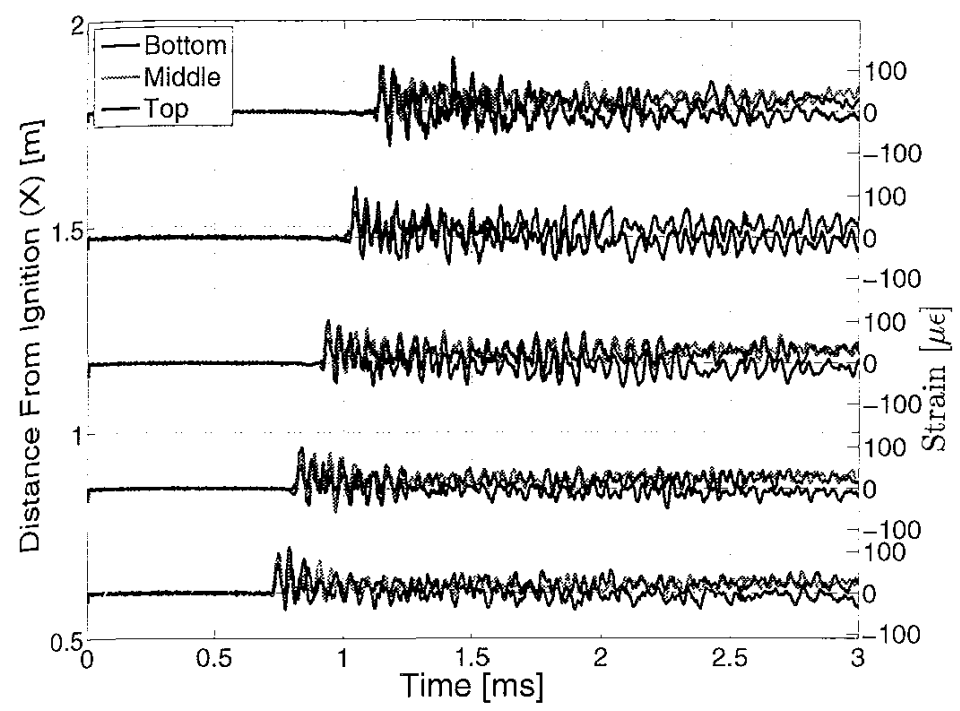

FIGURE 17. Strain traces (hoop direction) for a detonation with $h / d$ $=0.5$.

are confined to a smaller portion of the pipe. This leads to a reduction in the peak strains. At the deepest water levels, the DDT event is confined to such a small length that the strains respond as though to a detonation.

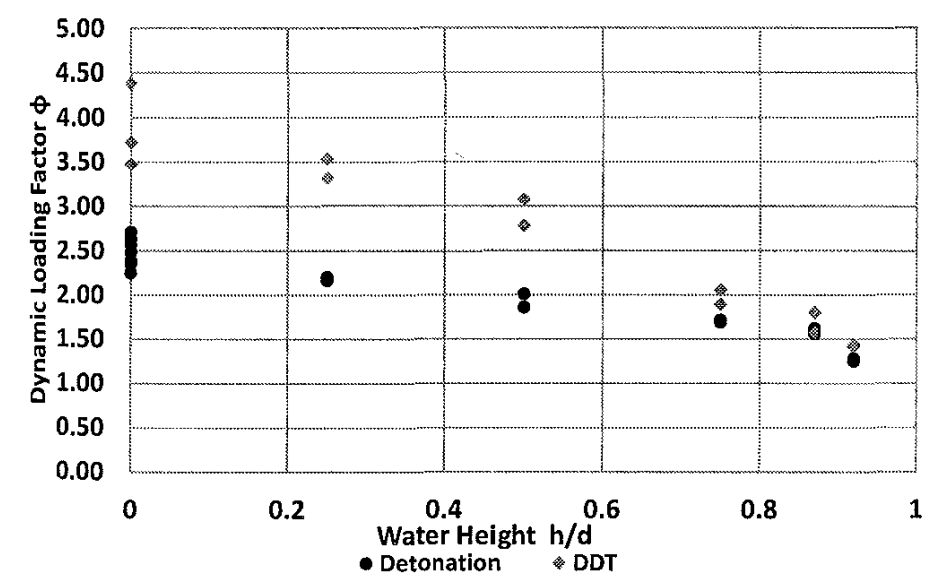

FIGURE 18. Dynamic loading factor vs. water depth for both detonations and DDT.

\section{Heat Transfer Effects}

In Figs. 16 and 17, the dynamic strain oscillations are superposed over a slowly varying strain offset of 30-50 $\mu \varepsilon$, which asymptotes to a constant over the first $5-10 \mathrm{~ms}$ and does not change significantly thereafter. This offset is shown more clearly in Fig. 19, where strain traces are plotted for a longer duration.

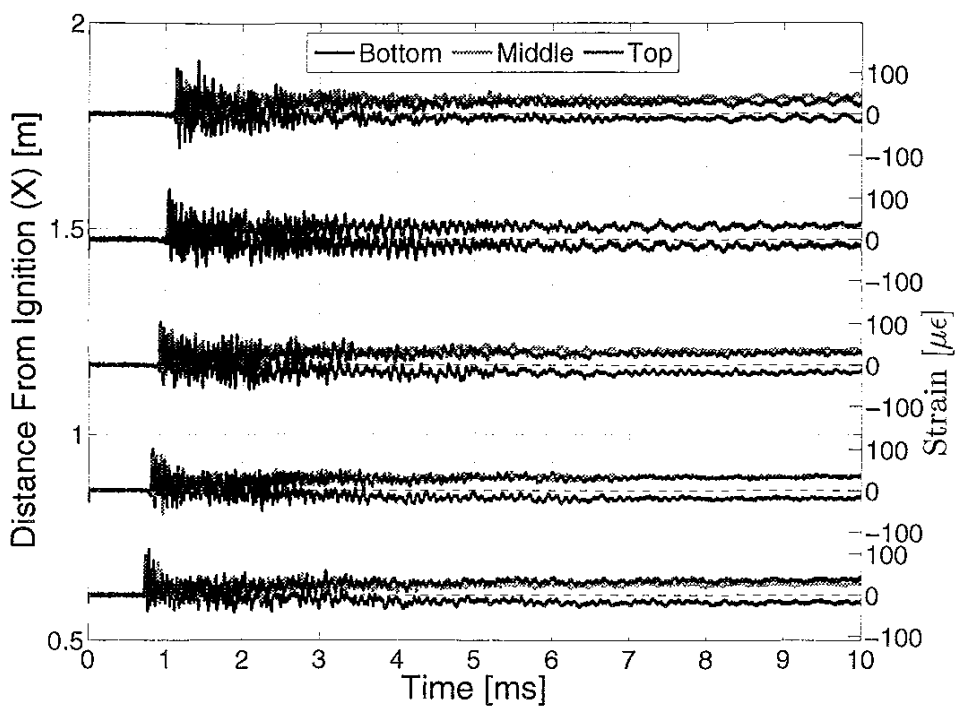

FIGURE 19. Strain trace for a detonation with water depth $h / d=$ 0.50 .

The strain offset is produced by a thin thermal layer in the tube wall, which stretches the rest of the tube through thermal expansion. A similar effect is analyzed in appendix D of [21] for tubes without water. Although the thickness of the thermal layer is quite small ( $\sqrt{\kappa t} \sim 0.1 \mathrm{~mm}$, or $\sim 3 \%$ of the wall thickness), the resulting strains can be non-negligible; in this experiment, thermal hoop strains were of the same order of magnitude as the dynamic strains.

The addition of water insulates the bottom of the pipe, locally eliminating the thermal layer. As a result, the bottom of the pipe is not stretched; in fact, there is slight compression due to bending caused by the non-axisymmetric temperature distribution.

Finite element computations were used to verify that these strain offsets are indeed thermal effects. A piecewise constant temperature distribution was assumed, as shown in the inset of Fig. 20. The thickness of the thermal layer was taken to be 0.1 $\mathrm{mm}$, which was obtained from $\sqrt{\kappa t}$ with $\kappa=4.2 \times 10^{-6} \mathrm{~m}^{2} / \mathrm{s}$ and $t$ $=5 \mathrm{~ms}$. The thermal layer was taken to be $70^{\circ} \mathrm{C}$ warmer than the rest of the pipe; this was the temperature that best matched the experimental data. The predictions of the finite element model are compared with experimental data in Fig. 20, where $\theta=0$ corresponds to the bottom of the pipe. The experimental strains are recorded after $100 \mathrm{~ms}$, long after the dynamic strain oscillations have died out. A sharp jump in hoop strain is observed at $\theta$ $=90^{\circ}$ and $270^{\circ}$, which is where the surface of the water touches the pipe wall, producing a large temperature gradient.

This thermal effect was also investigated experimentally by insulating a portion of the pipe with a thin sheet of neoprene. The neoprene sheet covered only the bottom half of the pipe, and was about $60 \mathrm{~cm}$ long so that it only affected strain gauges 


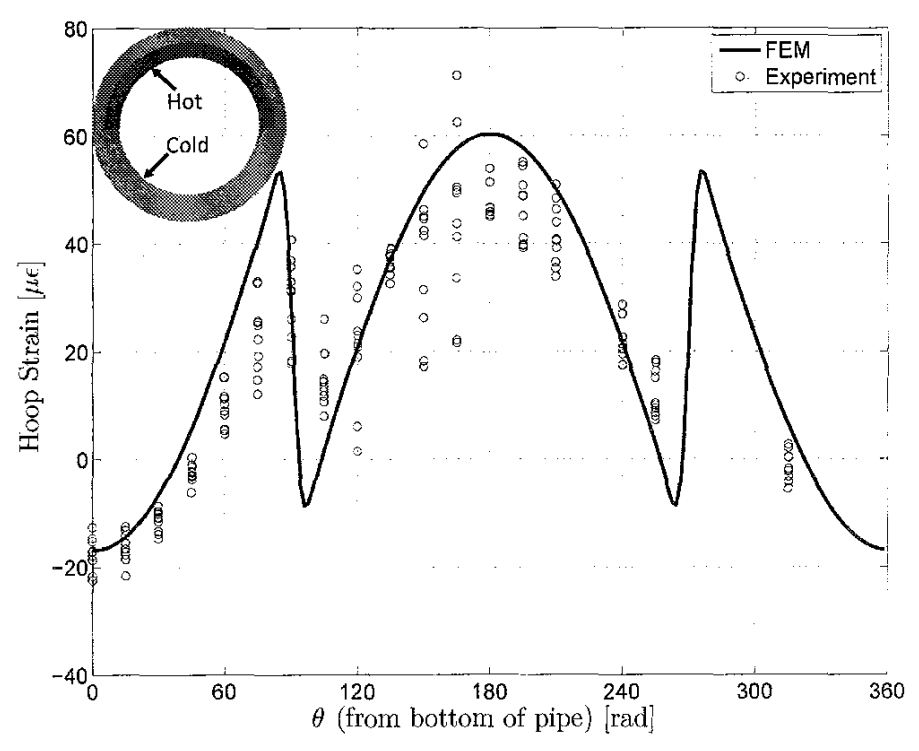

FIGURE 20. Thermal hoop strain as a function of angle. FEM: Finite element model assuming the temperature distribution shown in the inset diagram. Experiment: Hoop strains after $100 \mathrm{~ms}$ compiled from shots rotated at various angles.

S13-S18 (see Fig. 1). Strain traces from this configuration are shown in Fig. 21, where the bottom strain gauges at $\mathrm{x}=0.61$ and $0.86 \mathrm{~m}$ are insulated. Since there is no water in the pipe, the high frequency strain oscillations do not damp out as quickly. However, the slowly varying strain offset exhibits the same behavior as when the pipe was half filled with water: the top of the pipe expands while the bottom of the pipe remains unstretched or compresses slightly.

Another interesting feature of this plot is that the high frequency $(29 \mathrm{kHz})$ strain oscillations at the top, side, and bottom of the pipe are out of phase only for gauges that are insulated. This is similar to the lack of phasing that was observed in Fig. 17 for the water-filled case. One possible explanation is that the non-axisymmetric temperature distribution produced by either water or neoprene insulation slightly distorts the shape of the pipe and prevents it from oscillating in the axisymmetric hoop mode which would otherwise be dominant. For both water-filled and neoprene-insulated shots, power spectra of the strain traces show significant vibrational energy at freqencies of 3.4, 9.4, and $17.6 \mathrm{kHz}$. These frequencies are quite close to the first three natural frequencies $(3.2,8.9$, and $17.1 \mathrm{kHz})$ of what Blevins calls the radial-circumferential bending modes for an infinitely long tube [20]. Thus in addition to adding mass, the water modifies the vibrational response of the pipe by changing its shape through thermal expansion.

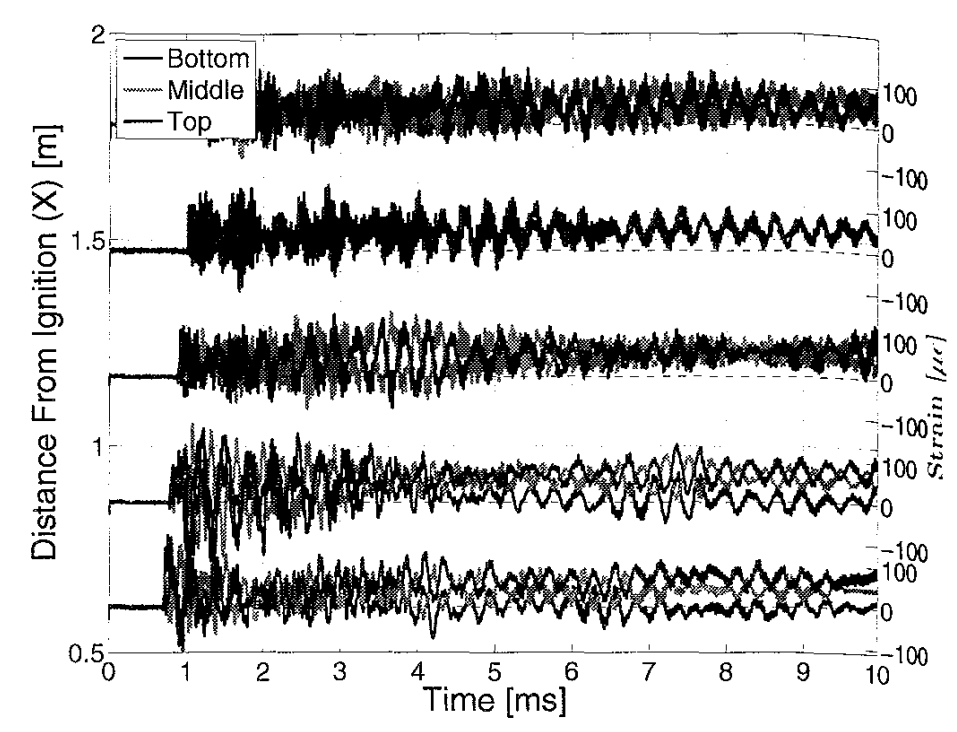

FIGURE 21. Strain traces for a detonation with a neoprene sheet covering the bottom half of the pipe at the first two strain measurement locations $(x=0.61$ and $0.86 \mathrm{~m})$.

\section{Conclusions}

Detonations passing over horizontal water layers in a cylindrical pipe were found to produce oblique shocks below the water that focus at the bottom of the pipe, generating peak pressures that are 4-6 times the peak detonation pressure. Peak pressures below the water did not show a clear dependence on water height, but above the water peak detonation pressures decreased by about $13 \%$ at the highest water height of 0.92 pipe diameters. Peak hoop strains also decreased systematically with water depth. Detonations and DDT were otherwise insignificantly affected by the presence of the water layer. Transition distances were consistently between 30 and $60 \mathrm{~cm}$, but more precise measurements of transition distance were not possible with the current apparatus. In all cases the detonation cell size was smaller than the gas layer height, so results may be different for other gas mixtures with larger cell sizes.

Strains due to thermal expansion were of the same order of magnitude as the dynamic strains. The water layer was found to insulate the bottom of the pipe, reducing thermal strains overall but producing increased thermal stresses where the water surface meets the pipe wall. The insulating effect of the water also appears to distort the pipe and modify its vibrational behavior.

\section{REFERENCES}

[1] Ciccarelli, G., and Dorofeev, S., 2008. "Flame acceleration and transition to detonation in ducts". Progress Energy Combust Sci, 34(4), pp. 499-550.

[2] Breitung, W., et al., 2000. Flame acceleration and deflagration-to-detonation transition in nuclear safety. 
Tech. rep., OECD Nuclear Energy Agency, August. Stateof-the-Art Report.

[3] Shepherd, J., 2009. "Structural response of piping to internal gas detonation". Journal of Pressure Vessel Technology, 131.

[4] Mahoney, L., Huckaby, J., Bryan, S., and Johnson, G., 2000. Overview of the flammability of gases generated in hanford waste tanks. Tech. Rep. PNNL-13269, U.S. Department of Energy, July.

[5] Shepherd, J. E., Akbar, R., and Rodriguez, E. A., 2009. "Gaseous detonation in piping systems partially filled with liquid". In ASME Pressure Vessels and Piping Conference, ASME. PVP2009-77734, July 26-30 2009, Prague, Czech Republic.

[6] Borisov, A., Kogarko, S., and Lyubimov, A., 1965. "Sliding of detonation and shock waves over liquid surfaces". Combustion, Explosion, and Shock Waves, 1(4), pp. 19-23.

[7] Teodorczyk, A., and Shepherd, J. E., 2012. Interaction of a shock wave with a water layer. Tech. Rep. FM2012-002, Graduate Aeronautical Laboratories, California Institute of Technology. Revised April 2012.

[8] Akbar, R., and Shepherd, J. E., 2010. Detonation initiation and propagation within gas layers in water-filled piping. Tech. Rep. FM2010-003, Graduate Aeronautical Laboratories California Institute of Technology, June. Revised June 2010.

[9] Kaneshige, M., and Shepherd, J., 1997. Detonation database. Tech. Rep. FM97-8, GALCIT, July.

[10] Fickett, W., and Davis, W., 1979. Detonation. University of California Press.

[11] Lee, J. H., 2008. The Detonation Phenomenon. Cambridge University Press.

[12] White, D. R., 1961. "Turbulent structure of gaseous detonation". Physics of Fluids, 4(4), pp. 465-480.

[13] Drazin, P., and Reid, W., 1981. Hydrodynamic Stability. Cambridge University Press.

[14] Inaba, K., and Shepherd, J. E., 2010. "Dynamics of cavitating flow and flexural waves in fluid-filled tubes subject to axial impact". In ASME Pressure Vessels and Piping Conference, ASME. PVP2010-25989, July 18-22, 2010. Bellevue, WA, USA.

[15] Ben-Dor, G., 2007. Shock Wave Reflection Phenomena, 2 ed. Springer.

[16] Skews, B., and Kleine, H., 2007. "Flow features resulting from shock wave impact on a cylindrical cavity". J. Fluid Mech, 580, pp. 481-493.

[17] Bitter, N., and Shepherd, J. E., 2012. An experimental study of detonation and transition to detonation in partially waterfilled pipes. Tech. Rep. FM2012-001, Graduate Aeronautical Laboratories, California Institute of Technology. Revised April 2012.

[18] Benedick, W., Knystautas, R., and Lee, J., 1985. "Dynam- ics of shock waves, explosions, and detonations". Progress in Astronautics and Aeronautics, 94, pp. 546-555.

[19] Whitham, G., 1974. Linear and Nonlinear Waves. John Wiley \& Sons.

[20] Blevins, R. D., 1979. Formulas for Natural Frequency and Mode Shape. Van Nostrand Reinhold Co.

[21] Shepherd, J. E., Karnesky, J., Pintgen, F., and Krok, J. C., 2008. Experimental measurements of strains and blast waves resulting from detonations in tubes. Tech. Rep. FM2006.010, Graduate Aeronautical Laboratories, California Institute of Technology. 